\title{
Test beam results from ATLAS electromagnetic calorimeter series modules
}

G. F. Tartarelli*i

Dipartimento di Fisica dell'Università di Milano and INFN, I-20133, Italy

E-mail: Francesco.Tartarelli@mi.infn.it

During the construction of the ATLAS EM calorimeter, four barrel modules and three endcap modules were exposed to beams of electrons, muons and pions from $10 \mathrm{GeV}$ to $245 \mathrm{GeV}$. Final results on position resolution and particle identification capabilities, linearity of energy response and resolution, and uniformity of energy response are presented.

International Europhysics Conference on High Energy Physics

July 21st - 27th 2005

Lisboa, Portugal

* Speaker.

${ }^{\dagger}$ On behalf of the ATLAS Electromagnetic Liquid Argon Calorimeter Group. 


\section{Introduction}

The electromagnetic calorimeter in the ATLAS experiment is a lead-liquid argon sampling calorimeter with accordion shaped absorbers and electrodes. Liquid argon technology has been chosen because of its intrinsic linear behaviour as function of the deposited energy, stability of the response and radiation tolerance.

The calorimeter is longitudinally segmented into three sections: strips, middle and back samplings. The strips sampling is segmented into narrow cells of size $\Delta \eta \times \Delta \phi \simeq 0.003 \times 0.1$ (in the end-cap, the segmentation of the first sampling is $\Delta \eta \times \Delta \phi \simeq 0.003-0.006 \times 0.1$ ). The other two compartments are segmented with towers of size $\Delta \eta \times \Delta \phi=0.025 \times 0.025$ (middle) and $\Delta \eta \times \Delta \phi=0.05 \times 0.025$ (back). A thin presampler detector is used to correct for energy losses in the dead material in front of the calorimeter. A detailed description of barrel and end-cap modules of the ATLAS electromagnetic calorimeter can be found in Refs [1].

A long test-beam program has been carried out along the years from early prototypes (19921996), to pre-series barrel and endcap modules (1998-2000) [1] to series modules. Four production modules (series numbers: P13, P15, M10 and M13) for the barrel calorimeter and three modules (ECC0, ECC1, ECC5) for the end-cap were tested in the CERN H6 and H8 beam lines during several months of tests during 2001-2002. The test setup is similar to the one described in [1]. The calorimeter performance was tested using secondary or tertiary electron and pion beams, with momenta from 20 to $245 \mathrm{GeV} / \mathrm{c}$ for barrel modules and from 20 to $150 \mathrm{GeV} / \mathrm{c}$ for end-cap modules.

\section{Energy resolution and linearity}

A calibration scheme based on a Monte Carlo study (which makes use of Geant 4) of the energy deposition in the active and inactive material of the calorimeter as well as of the material upstream and downstream of it has been developed [2]. In the model the energy measured in the presampler $\left(E_{P S}^{v i s}\right)$ and in the three layers of the calorimeter $\left(E_{i}^{v i s}\right)$ is weighted with factors (in general depending on the particle type, $\eta$, energy $E$ or shower depth $x_{l}$ ) which account for the energy lost in front of the active presampler layer (factors $a$ and $b$ ), the energy lost between the presampler and the calorimeter $(c)$, the calorimeter sampling fraction and the lateral leakage $(d)$, the longitudinal leakage $\left(f_{\text {leak }}\right)$ and the energy modulations along $\phi$ and $\eta$ due to the accordion shape $\left(f_{\text {eell }}\right)$ :

$$
E^{r e c}=\left[\left(a(E)+b(E) E_{P S}^{v i s}+c(E) \sqrt{E_{P S}^{v i s} E_{1}^{v i s}}+d(E) \sum_{i=1,3} E_{i}^{v i s}\right]\left(1+f_{\text {leak }}\left(x_{d}\right)\right) f_{\text {cell }}\right.
$$

An additional term $f_{\text {brem }}$, specific to the test beam set-up, is used to correct the mean energy for brem $\gamma$ 's lost in the passive material along the electron beam line. This MC scheme well describes the transverse and longitudinal shower profiles as well as the energy distributions in the various samplings. It also ensures good resolution and linearity as shown in Fig. 1. To measure the calorimeter linearity, in summer 2002 a dedicated set-up had to be used to precisely measure the beam energy. The linearity is better than $0.1 \%$ in the energy range $20-180 \mathrm{GeV}$. The sampling term of the energy resolution is measured to be $10 \%$, as expected, and the constant term measured on a single cell is about $0.20 \%$. 

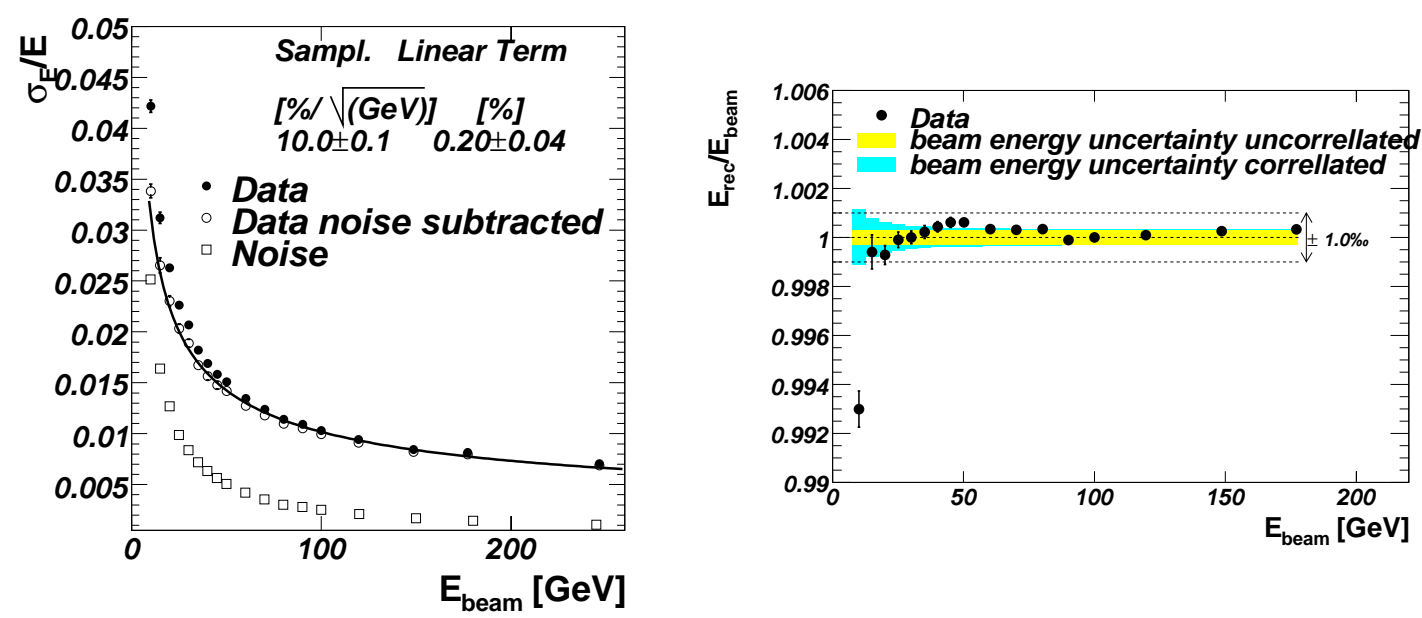

Figure 1: Left: fractional energy resolution versus beam energy for data before (closed circles) and after (open circles) noise subtraction. The noise contribution alone is also shown (open squares). Right: reconstructed electron energy to beam energy ratio versus beam energy. All points are normalized to the value measured at $\mathrm{E}=100 \mathrm{GeV}$ (barrel module $\mathrm{P} 13$ at $\eta=0.687$ and $\phi=0.282$ ).

For what the constant term is concerned, the goal, driven by the performance in physics channels (e.g., $H \rightarrow \gamma \gamma$ ), is to keep a $0.7 \%$ global constant term over the full calorimeter coverage for ATLAS precision physics. This will be achieved by assuring a $0.5 \%$ constant term on a region $\Delta \eta \times \Delta \phi=0.2 \times 0.4$ by construction and thanks to electronic calibration and offline corrections. Then these regions (440 regions on the whole calorimeter) will be intercalibrated in situ at the $0.3 \%$ level using $Z \rightarrow e e$ events.

To check the assumption at the basis of this strategy, a cell-by-cell position scan of whole modules at fixed energy has been performed. The uniformity of the calorimeter response is shown in Fig. 2. Each point corresponds to the peak of the energy distribution of events impinging in one cell of the middle compartment, as a function of $\eta$ for various $\phi$ positions. The response dispersion is less than $0.5 \%$ in the required $\Delta \eta \times \Delta \phi=0.2 \times 0.4$ region and less than $0.6 \%$ in a region about six time larger (corresponding to a full module).

\section{Position resolution}

The transverse and longitudinal segmentation of the calorimeter has been optimized for particle ID and angular measurement with a key role played by the first sampling of $\eta$-strips.

At the LHC the beam spread in the $z$ coordinate is about $5.6 \mathrm{~cm}$ (while it is very good, about $15 \mu \mathrm{m}$, in the $x-y$ plane). The beam spread contributes to the angular resolution of the $H \rightarrow \gamma \gamma$ invariant mass. The calorimeter segmentation also allows a stand-alone reconstruction of the shower direction and vertex position using the shower barycenter mesurement in the strips and middle compartments. A position resolution of about $250 \mu \mathrm{m}(550 \mu \mathrm{m})$ in the strips (middle) compartment was estimated at low $\eta$, after unfolding the resolution (about $250 \mu \mathrm{m}$ ) of four multi-wire proportional chambers used in the test beam to determine the beam impact point at the calorimeter [3].

Fig. 3 shows the resolution on the polar angle $\theta$ obtained with this method. The polar angle resolution is shown as a function of $\eta$ in the barrel compartment and as a function of the beam 

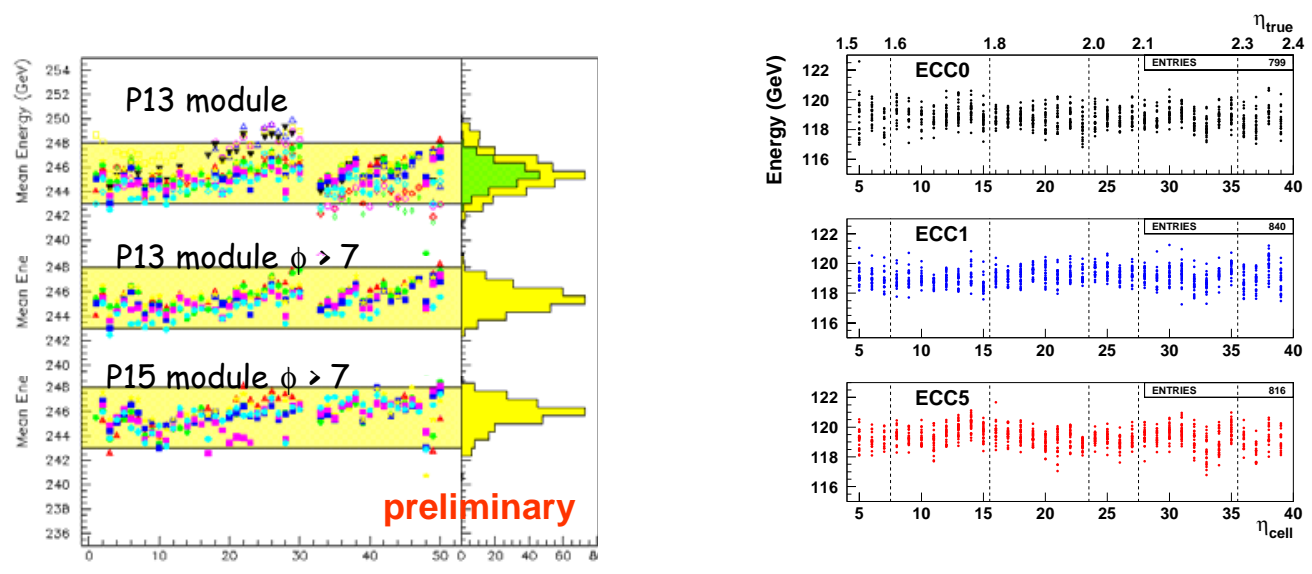

Figure 2: Calorimeter energy response as a function of $\eta$ for various $\phi$ position for two barrel (left) and three endcap (right) modules at fixed energy (245 GeV in the barrel and $120 \mathrm{GeV}$ in the endcap).
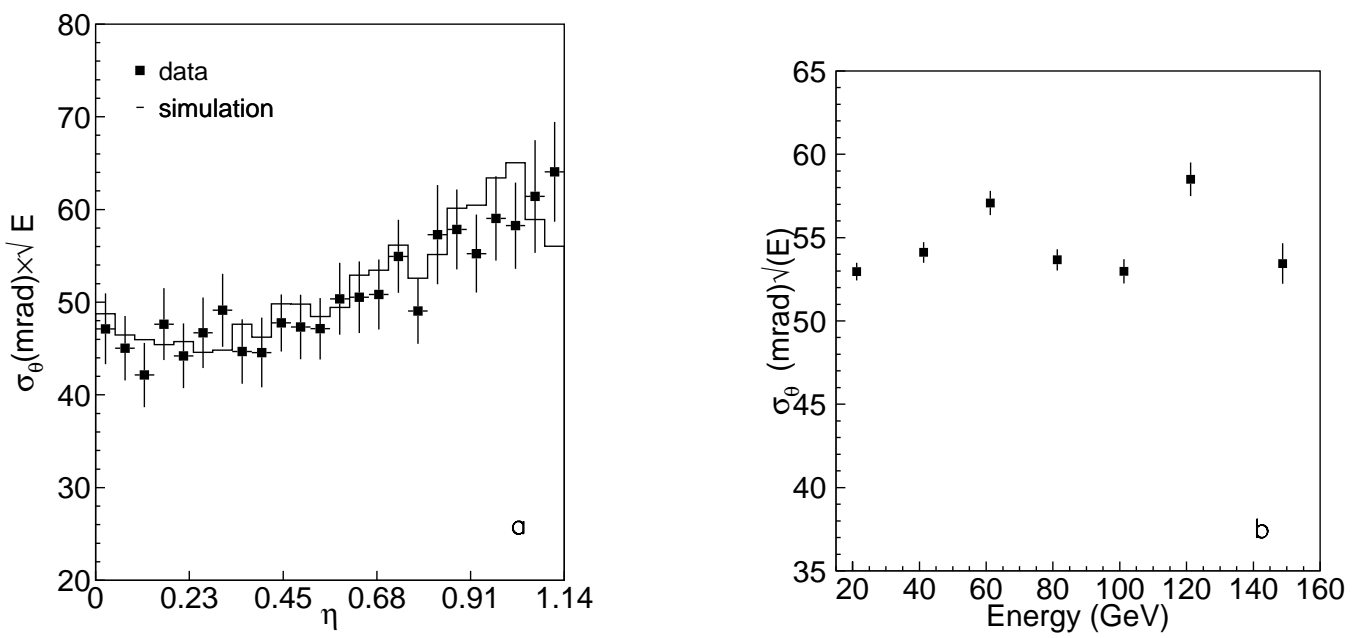

Figure 3: Angular resolution in polar angle $\theta$ times the square root of the beam energy as a function of $\eta$ (barrel module P13 at $\phi=0.26$ and $\mathrm{E}=245 \mathrm{GeV}$ ) and as function of the beam energy (endcap module ECC1 at $\eta=1.74$ and $\phi=0.18 \mathrm{rad})$.

energy in the endcap. It can be shown that the result obtained, $\sigma_{\theta}=50-60 \mathrm{mrad} / \sqrt{E(\mathrm{GeV})}$, is in good agreement with the design expectation and is enough to keep the contribution of the angular resolution in the $H \rightarrow \gamma \gamma$ channel less than the photon energy resolution term.

\section{References}

[1] B. Aubert et al., Nucl. Instrum. Meth. A 500 (2003) 178-201.

B. Aubert et al., Nucl. Instrum. Meth. A 500 (2003) 202-231.

[2] Energy Linearity and Resolution of the ATLAS Electromagnetic Barrel Calorimeter at an Electron Test beam, to be published in NIM.

[3] J. Colas et al., Nucl. Instrum. Meth. A550 (2005) 96-115. 